\title{
Percepción de alteraciones dermatológicas de baja expresión y calidad de vida: el papel mediador del afrontamiento*
}

\author{
Perception of Low-Level Expression Dermatological Diseases \\ and Quality of Life: Moderating Role of Coping
}

Recibido: 19 de diciembre de 2013 | Revisado: 27 de octubre de 2014 | Aceptado: 5 de febrero de 2015

\author{
ESTEFANÍA HERNÁNDEZ-FERNAUD ** \\ BERNARDO HERNÁNDEZ *** \\ CRISTINA RUIZ**** \\ Universidad de La Laguna, Canarias, España
}

doi:10.11144/Javeriana.upsy14-2.padb

Para citar este artículo: Hernández-Fernaud, E., Hernández, B., \& Ruiz, C. (2015). Percepción de alteraciones dermatológicas de baja expresión y calidad de vida: el papel mediador del afrontamiento. Universitas Psychologica, 14(2), 523-534. http:// dx.doi.org.10.11144/Javeriana.upsy14-2.padb

Los autores agradecen las sugerencias y comentarios de los revisores anónimos de este trabajo. Artículo de investigación.

** Profesora Titular de Universidad, Departamento de Psicología Cognitiva, Social y Organizacional. Contacto principal para correspondencia editorial. Correo electrónico: efernaud@ull.es

*** Catedrático de Universidad, Departamento de Psicología Cognitiva, Social y Organizacional. Correo electrónico: bhdezr@ull.edu.es

***** Profesora Ayudante Doctor, Departamento de Psicología Cognitiva, Social y Organizacional. Correo electrónico: cruizpa@ull.edu.es

\section{RESUMEN}

La percepción de una enfermedad tiene que ver con las estrategias de afrontamiento de la misma y la calidad de vida del paciente. El objetivo de este trabajo es contrastar el papel mediador de varias estrategias de afrontamiento en la relación entre la percepción de la enfermedad dermatológica y la calidad de vida. Participaron 220 personas que cumplimentaron, de forma individual y anónima, un cuestionario que evaluaba expresión sintomatológica cutánea, impacto psicológico de las condiciones de la piel, estrategias de afrontamiento ante alteraciones cutáneas y calidad de vida. Los resultados muestran que, en personas que creen tener una alteración dermatológica leve, la influencia del impacto psicológico de las condiciones de la piel sobre la calidad de vida está mediada de forma diferencial por las estrategias de afrontamiento. Mientras que el uso de la estrategia de pensamientos positivos contribuye a mantener la calidad de vida, utilizar la estrategia pensamientos desiderativos produce una disminución de la misma. Palabras clave

afrontamiento; análisis mediacional; calidad de vida; percepción de enfermedad; problemas dermatológicos

\footnotetext{
A B S T R A C T

The perception of the disease is related to coping strategies and quality of life. The purpose of this paper is to analyze the mediating role of specific coping strategies in the influence of the perception of low-level expression dermatological diseases on quality of life. Two hundred-twenty participants completed, individually and anonymously, a questionnaire about symptoms, psychological impact, coping strategies and quality of life. Results show that, in people withperception of low-level expression dermatological diseases, the effect of the psychological impact to skin conditions on the quality of life is mediated differentially by coping strategies. While the Positive thinking strategy contributes to maintain the quality of life, the Wishful thinking strategy produces a decrease of the same.

Keywords

coping; illness perception; mediational role; quality of life; skin problems
} 


\section{Introducción}

La mayoría de las personas considera que la salud es un elemento clave en su calidad de vida (Delhey, 2004), especialmente cuando se padece una enfermedad con sintomatología severa (Dijkers, 2005; Pantiga, C., López, L., Pérez, M., Rodríguez, M., Linares, A., Dieguez, L. G., ... \& Rodrigo, L. 2005). Esta aseveración también se aplica a los problemas dermatológicos. Así, diversos estudios han encontrado que el estado de la piel influye considerablemente en los hábitos diarios, la autoestima y la calidad de vida (Badía, Mascaró, \& Lozano, 1999; Drake, Patrick, Fleckman, André, Baran, Haneke, ... Tosti, 1999; Niemeier et al., 1998).

Además, aun cuando estos problemas dermatológicos no presenten manifestaciones severas suelen producir secuelas psicológicas y sociales importantes, como ansiedad, estrés y/o depresión (Gupta, Gupta, \& Watteel, 1997; Rubino, Sonnino, Pezzarossa, Ciani, \& Bassi, 1995). No obstante, no toda la investigación verifica esta relación. Por ejemplo, Nichol, Margolis, Lippa, Rower y Quell (1996) y Sampogna, Sera, Aveni e Istituto Dermopatico dell'Immacolata (IDI) Multipurpose Psoriasis Research on Vital Experiences (IMPROVE) investigators (2004) señalan la ausencia de relación o la existencia de relaciones negativas entre la enfermedad y la calidad de vida de los pacientes.

Una razón para esta discrepancia está en las diferentes operacionalizaciones sobre calidad de vida que se han utilizado en los estudios. Así, en unos casos se evalúa el impacto de la enfermedad en la calidad de vida en términos generales, mientras que en otros se considera el efecto sobre la calidad de vida relacionada con la salud. La calidad de vida se entiende como un conjunto de experiencias relacionado con el bienestar general, que tiene un claro componente subjetivo al partir de las expectativas personales e incluir una valoración global sobre la vida y que trasciende a la salud (Revicki, Osoba, Fairclough, Barofsky, Berzon, Leidy, \& Rothman, 2000).

La calidad de vida relacionada con la salud ha sido definida como las reacciones y percepciones que tienen los pacientes con respecto a su salud, refiriéndose a dominios físicos, funcionales, psicológicos y sociales. Estos dominios pueden ser evaluados tanto a nivel objetivo como subjetivo, siendo la percepción subjetiva de la enfermedad lo que determina la calidad de vida experimentada en relación con la salud (Rodríguez-Marín, Pastor, \& López-Roig, 1993).

Hernández-Fernaud, Hernández, Ruiz, Rodríguez y Betancor (2008) hallaron que las enfermedades dermatológicas de baja expresión sintomatológica tenían un impacto claro en medidas de calidad de vida relacionadas con la salud, pero no sobre medidas de calidad de vida en términos de bienestar y satisfacción con la vida. En este mismo sentido, Nichol et al. (1996) encontraron que las enfermedades dermatológicas con baja expresión afectan la calidad de vida cuando se utilizan medidas relacionadas con las emociones, los sentimientos y la autoimagen.

A ese escenario de incertidumbre se suma la duda sobre si la severidad y la duración de la enfermedad se relacionan directamente con la calidad de vida (Drake et al., 1999; Mazzotti, Picardi, Sampogna, Sera, Pasquín, \& Abeni. 2003), o si, esa relación está mediada por otras variables, entre las que destacan la percepción de la enfermedad y las estrategias de afrontamiento (Schmid-Ott et al., 2005).

La percepción de la enfermedad juega un papel fundamental en el tipo de valoraciones que los enfermos hacen de su sintomatología, la relación con los profesionales de la salud, la respuesta y adhesión al tratamiento y la reacción ante los síntomas que experimentan y perciben (Benyamini, Goner-Shilo, \& Lazarov, 2012; Karademas, Zarogiannos, \& Karamvakalis, 2010; Vinaccia, Quiceno, \& Remor, 2012). En este sentido, el Modelo de la Autorregulación establece que la enfermedad adquiere sentido y significado para la persona a partir de la representación que se hace de la misma (Leventhal, Leventhal, \& Cameron, 2001). El afrontamiento se refiere a las estrategias utilizadas por las personas para manejar las demandas de una situación estresante, así como a la manera de gestionar las emociones asociadas a dicho suceso (Lazarus \& Folkman, 1986; Nava-Quiroz, Vega-Valero, \& So- 
ria, 2010). El afrontamiento se entiende como un proceso dinámico en el que se activan distintas estrategias en función de los cambios en el entorno y de la percepción de la situación (Lazarus, 1993; Rodríguez-Marín, Pastor, \& López-Roig, 1993).

La relación entre el afrontamiento de los problemas dermatológicos y la calidad de vida ha sido puesta de manifiesto en diversos estudios. Por ejemplo, Rapp, Cottrell y Leary (2001) señalan que las habilidades sociales de afrontamiento son un factor determinante del impacto de la psoriasis sobre la calidad de vida. Wahl, Hanestad, Wiklund y Moum (1999) encuentran que los pacientes que utilizan estrategias centradas en el optimismo y la normalización muestran mayor grado de salud mental, y que aquellos que utilizan estrategias emotivas de afrontamiento presentan más problemas relacionados con la salud y el bienestar.

Con una metodología centrada en comprobar el efecto del tiempo en el uso de las estrategias de afrontamiento, Scharloo, Kaptein, Weinman, Bergman, Vermeer, \& Rooijmans, (2000) llevan a cabo un estudio longitudinal de 69 pacientes con psoriasis. De acuerdo con sus observaciones, los pacientes que al principio desarrollan afrontamiento caracterizado por una mayor expresión de emociones, búsqueda de apoyo social y distracción y un menor grado de adaptación pasiva, necesitan un número inferior de sesiones de terapia, están menos ansiosos y deprimidos y tienen una mejor salud física un año más tarde. Fortune, Richards, Griffihs y Main (2002) encuentran que el afrontamiento modula la respuesta de ansiedad frente a la psoriasis y se relaciona con la preocupación por la enfermedad, pero también encuentran que el afrontamiento tiene una relación insignificante con las evaluaciones de estrés y gravedad de la psoriasis.

También Van Loey y Van Son (2003) obtienen que cuando los pacientes con cicatrices de quemaduras utilizan un estilo de afrontamiento centrado en la emoción y la evitación, y poco centrado en la aceptación tienen mayor cantidad de síntomas de depresión, estrés postraumático y estrés en general. Cuando utilizan estilos de afrontamiento más centrados en la solución del problema y la búsqueda de apoyo social, la calidad de vida se reduce en menor
medida.Asimismo, se ha observado que el grado de severidad en casos de alopecia no es el único factor que determina la calidad de vida de los pacientes, sino que también influyen los estilos de afrontamiento. El daño subjetivo de estos pacientes no es una función primaria de la alopecia, sino resultado de un afrontamiento desadaptativo (Schmidt, Fischer, Chren, Strauss, \& Elsner, 2001).

Aunque parece claro que la relación entre patología cutánea y bienestar general de los pacientes está condicionada por la forma en que estos aborden su problema de salud, no han quedado establecidas qué estrategias concretas de afrontamiento están implicadas en esta relación en alteraciones leves. En este sentido, el objetivo de esta investigación es evaluar el papel de distintas estrategias de afrontamiento en la relación entre la percepción de una enfermedad dermatológica de baja expresión y la calidad de vida. Debido al posible solapamiento conceptual y a la multicolinealidad que pueda haberse producido en parte de la investigación anterior al relacionar medidas de calidad de vida relacionada con la salud y la calidad de vida, la relación entre estas variables podría esclarecerse si se utilizaran medidas basadas en una valoración en términos de satisfacción con la vida, más que en términos de bienestar subjetivo o psicológico.

Nuestra hipótesis es que las estrategias de afrontamiento mediarán de forma diferencial el impacto de la percepción de la enfermedad dermatológica sobre la calidad de vida. Concretamente, se espera que el uso de estrategias de aproximación al problema favorezca una mayor calidad de vida, mientras que el uso de estrategias de evitación tendrá el efecto contrario.

\section{Método}

\section{Participantes}

El cuestionario se aplicó inicialmente a 292 personas que informaron sobre el estado de su piel. De estas, se eliminaron 72 por carecer de sintomatología dermatológica objeto de esta investigación. Por tanto, la muestra fue de 220 personas residentes en las Islas Canarias con edades comprendidas 
entre 19 y 82 años, con una media de 31.23 años $(D E=14.28)$. El $66.36 \%$ de la muestra correspondió a mujeres y el $33.63 \%$ a varones. El $50.7 \%$ lo conformaban estudiantes universitarios, el $37.3 \%$ desempeñaba alguna actividad laboral y el $11.9 \%$ restante estaba compuesto por desempleados, jubilados y amas de casa. Excepto en el caso de los estudiantes universitarios, el resto de la muestra se obtuvo mediante un muestreo incidental de cuotas por género e intervalos de edad.

\section{Diseño}

Se ha utilizado un diseño transversal, donde la variable criterio es la calidad de vida y las variables predictoras son la expresión sintomatológica cutánea y el impacto psicológico de las condiciones de la piel. Además, se incluyen diferentes estrategias de afrontamiento como variables mediadoras.

\section{Instrumentos}

Se utilizó un cuestionario compuesto por cuatro instrumentos escalares. El primer instrumento identificaba la sintomatología dermatológica percibida y cuantificaba el grado de expresión percibido de las alteraciones dermatológicas y comportamientos relacionados con dichas alteraciones. El segundo instrumento evaluaba el impacto psicológico que tiene la alteración dermatológica. El tercer instrumento valoraba las estrategias de afrontamiento utilizadas para enfrentarse a la alteración dermatológica indicada. Por último, se utilizó un instrumento para evaluar la calidad de vida entendida como satisfacción específica con diferentes aspectos de la vida. Se elaboraron tres versiones de la batería, que variaban en el orden de presentación de los instrumentos.

\section{Escala de Identificación y Expresión de Sintomatología Cutánea}

Esta escala está compuesta por un listado de 26 alteraciones dermatológicas (Hernández-Fernaud, Hernández, Ruiz, \& Ruiz, 2009). La inclusión de las distintas alteraciones dependió de su frecuencia de aparición, la facilidad para detectar su presencia y de que, en su manifestación más agresiva, aun no suponiendo una patología grave, afectara la vida de las personas. Los participantes debían indicar en una escala de seis puntos en qué medida, en el último mes, habían tenido cada una de ellas. El autoinforme sobre enfermedades de la piel ha mostrado ser un procedimiento de diagnóstico y de investigación válido y fiable, comprobándose que las valoraciones de los pacientes coinciden en un alto porcentaje con un examen clínico posterior (Mazzoti et al., 2003; Rapp, Cottrell, \& Leary, 2001).

Posteriormente, los participantes debían seleccionar aquella alteración que más condicionara su vida y contestar, en una escala tipo Likert de cinco puntos (variando las etiquetas de cada anclaje en función de la pregunta), a nueve preguntas sobre sus síntomas (p. ej., daño general, dolor, picor, ardor, sangre, irritación). Con estos ítems se obtuvo una puntuación media que indicaba la expresión sintomatológica de la alteración. La consistencia interna de esta escala fue 0.81 (Hernández-Fernaud et al., 2008).

\section{Escala de Impacto Psicológico de las Condiciones de la Piel}

Se seleccionaron y adaptaron 13 ítems del cuestionario Skindex-61, referidos a cómo y con qué intensidad los pacientes experimentan sentimientos y emociones respecto al estado de su piel (Hernández-Fernaud et al., 2008). El cuestionario Skindex evalúa calidad de vida relacionada con la salud a nivel funcional, emocional y sintomático. Este instrumento ha mostrado un buen nivel de fiabilidad, validez y sensibilidad (Chren, Lasek, Quinn, Mostow, \& Zyzanski, 1996; Jones-Caballero, Penas, García-Diez, Chren, \& Badía, 2002). Para esta investigación, se seleccionaron ítems del área emocional que valoraban el grado de acuerdo de los participantes con frases como "Estoy enfadado con el estado de mi piel", "Mi piel hace que me sienta marginado" o "El estado de mi piel hace que me sienta deprimido". Los participantes debían indicar, en una escala de 5 puntos $(1=$ nunca; $5=$ siempre), en qué medida habían experimentado, en el último mes, cada una de las situaciones descritas. 
Una puntuación alta indica el mayor impacto psicológico que tienen las condiciones de la piel sobre la persona. Esta escala obtuvo un alfa de Cronbach de 0.9 (Hernández-Fernaud et al., 2008). Para determinar su validez se siguió el procedimiento de known-group validity (Hernández-Fernaud et al., 2009; Zeller \& Carmines, 1980), separando los grupos en función de las respuestas a una pregunta sobre el daño físico producido por la alteración dermatológica en la piel. Los resultados pusieron de manifiesto que efectivamente existen diferencias estadísticamente significativas en el impacto psicológico de las condiciones de la piel en los grupos extremos de la variable daño físico en la piel $\left(F_{(1,}\right.$ $\left.{ }_{189)}=19.69, p<0.001\right)$.

\section{Cuestionario de Estrategias de Afrontamiento ante Alteraciones Cutáneas}

Este instrumento, elaborado por HernándezFernaud et al. (2009), evalúa las estrategias de afrontamiento que utiliza la persona para enfrentarse a las alteraciones de la piel, mediante 29 ítems que se agrupan en seis subescalas: 1) Pensamientos desiderativos: expresa deseos de que la situación no hubiera ocurrido y/o tuviera consecuencias diferentes, y sentimientos de tristeza y culpa. Algunos ítems de esta escala son: "He deseado que las cosas hubieran sido de otra manera" o "He deseado poder cambiar lo que me ha ocurrido". El índice de consistencia interna fue de $\alpha=0.87$; 2) Búsqueda de soluciones: se refiere a estrategias orientadas a la búsqueda de ayuda profesional para la solución del problema y a la planificación. Algunos ítems de esta escala son: "He realizado seguimientos médicos de forma regular" y "He buscado ayuda profesional". El índice de consistencia interna fue $\alpha=0.9 ; 3$ ) Búsqueda de apoyo social: incluye acciones de acercamiento a otras personas para solucionar el problema o sentirse mejor. La consistencia interna fue $\alpha=0.82$. Algunos ejemplos de ítems son: "Pido a parientes y amigos que me ayuden a pensar en el problema" y "He hablado con alguien sobre cómo me siento"; 4) Búsqueda de información: se refiere a estrategias de búsqueda de información y comprensión del problema. Los ítems se refieren a aspectos como "He intentado mejorar mi información sobre el problema" u "Oigo radio, veo televisión o leo acerca del problema". El índice de consistencia interna fue $\alpha=0.83$; 5) Pensamientos positivos, expresa la consideración de aspectos positivos de la situación. La consistencia interna fue $\alpha=0.76$. Algunos ejemplos de ítems son: "Me he concentrado en las cosas buenas de mi vida" o "Intento ver los aspectos positivos de la situación" y 6) Distanciamiento: incluye las estrategias de relativización del problema, de modo que este no interfiera en otros ámbitos de la vida. Algunos ítems de la escala son "Procuro que el problema no interfiera en otros aspectos de mi vida" y "Trato de mantener la calma". La consistencia interna fue $\alpha=0.67$. La validez se puso a prueba mediante un procedimiento de known-group validity resultando satisfactoria (Hernández-Fernaud et al., 2009). Los participantes indicaban, en una escala Likert de cinco puntos $(1=$ nunca; $5=$ siempre), con qué frecuencia utilizaban cada forma de afrontamiento.

\section{Escala de calidad de vida entendida como satisfacciones específicas}

La Escala de Calidad De Vida (Quality of Life Scale [QOLS]) fue diseñada por Flanagan (1978) y posteriormente modificada por Burckhardt y Anderson (2003). La QOLS proporciona una medida de la calidad de vida en sentido amplio (no solo relacionada con la salud), mediante la evaluación de la satisfacción con aspectos específicos o situacionales (por ejemplo, la salud, las relaciones con familiares, la actividad laboral). Para este estudio, se añadieron, a las16 situaciones originales, otras cinco que recogían aspectos considerados relevantes en la literatura (relaciones con los vecinos, actividades en la naturaleza, viajar, labores domésticas y actividades manuales). Se responde en una escala Likert de cinco puntos (1= muy insatisfecho; 5= muy satisfecho), donde una puntuación alta representa mayor calidad de vida. La fiabilidad de esta escala fue de 0.84 (Hernández-Fernaud et al., 2008). Su validez ha sido puesta a prueba por Burckhardt y Anderson 
(2003), Burckhardt, Anderson, Archenholtz y Hägg (2003), y en su versión española por Latorre-Román, Martínez-Amat, Martínez-López, Moral, Santos, \& Hita-Contreras, (2014).

\section{Procedimiento}

Los participantes cumplimentaron el cuestionario de forma individual; aproximadamente, la mitad de la muestra en sesiones grupales de clase, el resto en su domicilio con el apoyo de entrevistadores entrenados. La cumplimentación del cuestionario tuvo una duración aproximada de 25 minutos.

Dado que este trabajo no implicaba riesgo para los participantes, se solicitó el consentimiento oral informado y estos fueron informados de que su colaboración en esta investigación era voluntaria y anónima. Se garantizó la confidencialidad de los datos obtenidos y el tratamiento colectivo de los mismos.

\section{Análisis estadísticos}

Antes de iniciar el análisis mediacional se comprobó la existencia de outliers multivariados con la distancia de Mahalanobis y la multicolinearidad mediante el nivel de tolerancia (Tabachnick \& Fidell, 1989).

A continuación, se analizaron los datos mediante el procedimiento de regresión múltiple para la comprobación del efecto de mediación desarrollado por Baron y Kenny (1986), donde la variable criterio fue calidad de vida y las variables predictoras eran impacto psicológico de las condiciones de la piel y los seis factores de estrategias de afrontamiento ante alteraciones cutáneas.

El procedimiento para realizar un análisis mediacional mediante regresión incluye tres pasos (Baron \& Kenny, 1986; Judd \& Kenny, [1981] 2010). El primer paso consiste en demostrar que la variable predictora está relacionada significativamente con la/s variable/s mediadora/s, por lo que se evaluó en qué medida el impacto psicológico de las condiciones de la piel explica las estrategias de afrontamiento. El segundo paso radica en demostrar que la variable predictora está relacionada signifi- cativamente con la variable criterio, así se estimó la regresión del impacto psicológico de las condiciones de la piel sobre la calidad de vida. Por último, debe comprobarse el efecto de la variable predictora y las mediadoras sobre la variable criterio, para ello se realizó un análisis de regresión del impacto psicológico y las estrategias de afrontamiento sobre la calidad de vida. En este último paso, debe comprobarse que la magnitud de la relación de la variable predictora con la variable criterio es menor cuando se incluye la variable mediadora. Para calcular la significación estadística de este efecto se utilizó el test de Sobel (Sobel, 1982).

\section{Resultados}

La inclusión en el análisis mediacional de los participantes en el estudio se realizó mediante las puntuaciones de la Escala de Identificación y Expresión de Sintomatología Cutánea. Para ello, se calcularon los cuartiles de dicha variables, y se eliminaron los participantes que se encontraban en el primer cuartil $(n=72)$, ya que carecían de molestias físicas. De modo que se excluyó del análisis posterior a los participantes que aun teniendo alguna patología cutánea no presentaban molestias físicas, y se incluyó a quienes sí presentaban alguna molestia física de baja expresión. Las puntuaciones obtenidas en la Escala de Identificación y Expresión de Sintomatología Cutánea pusieron también de manifiesto que el resto de los participantes obtuvo valores de expresión sintomatológica que oscilaban desde 1.22 a 3.4, siendo la media $1.69(D E=0.44)$. Entre las alteraciones dermatológicas identificadas, aquellas que condicionaban la vida en mayor medida fueron la presencia de granos o espinillas en la cara, la sensibilidad de la piel al sol, la celulitis, la caída del pelo, la sequedad de la piel y tener caspa y/o grasa en el cuero cabelludo.

Antes de realizar el análisis, se comprobó si la distribución de las variables era normal. El estadístico Kolmogorov-Smirnov fue significativo en todas las variables excepto en calidad de vida. Este nivel de significación puede deberse al tamaño de la muestra, por lo que se consideró el tamaño del estadístico que en ningún caso fue superior a 0.3, 
PeRCEPCIÓN DE ALTERACIONES DERMATOLÓGICAS DE BAJA EXPRESIÓN Y

CALIDAD DE VIDA: EL PAPEL MEDIADOR DEL AFRONTAMIENTO

TABLA 1

Estadísticos descriptivos, estadístico de normalidad Kolmogorov-Smirnov (KS) y matriz de correlaciones entre las variables

\begin{tabular}{|c|c|c|c|c|c|c|c|c|c|c|c|}
\hline & Variables & M & $D E$ & KS & 1 & 2 & 3 & 4 & 5 & 6 & 7 \\
\hline 1 & Impacto psicológico & 1.58 & 0.56 & $0.17 * * *$ & & & & & & & \\
\hline 2 & P. desiderativos & 2.40 & 1.00 & $0.09 * * *$ & $0.51 * *$ & & & & & & \\
\hline 3 & B. soluciones & 2.61 & 1.20 & $0.10 * * *$ & $0.23 * *$ & $0.38 * *$ & & & & & \\
\hline 4 & B. ароуо & 2.56 & 0.95 & $0.08 * *$ & $0.21 * *$ & $0.46 * *$ & $0.65 * *$ & & & & \\
\hline 5 & B. información & 2.56 & 1.01 & $0.09 * * *$ & $0.40 * *$ & $0.52 * *$ & $0.62 * *$ & $0.61 * *$ & & & \\
\hline 6 & P. positivos & 2.76 & 0.91 & $0.07 * * *$ & $0.19 * *$ & $0.37 * *$ & $0.42 * *$ & $0.58 * *$ & $0.51 * *$ & & \\
\hline 7 & Distanciamiento & 3.07 & 0.87 & $0.11 * * *$ & 0.13 & $0.21 * *$ & $0.36 * *$ & $0.45 * *$ & $0.37 * *$ & $0.51 * *$ & \\
\hline 8 & Calidad de vida & 3.37 & 0.53 & 0.05 & $-0.28 * *$ & $-0.29 * *$ & 0.08 & 0.06 & -0.02 & 0.09 & 0.02 \\
\hline
\end{tabular}

$* p \geq 0.05 . * * p \geq 0.01$. *** $p \geq 0.001$.

Fuente: elaboración propia

por lo que se asume la distribución normal de las variables. También se comprobó que se cumplían los supuestos de ausencia de outliers multivariados, con la distancia de Mahalanobis y de multicolinearidad, mediante el nivel de tolerancia (Tabachnick $\&$ Fidell, 1989). En la Tabla 1 se presentan los estadísticos descriptivos para las variables implicadas en el análisis, así como la prueba de normalidad y las correlaciones entre ellas. También se comprobó la independencia de los residuos con el estadístico Durbin-Watson, que se ha incluido en la Tabla 2.

A continuación, se procedió a realizar el análisis mediacional mediante regresión (Baron \& Kenny, 1986). Primero, se evaluó en qué medida el impacto psicológico explica las distintas estrategias de afrontamiento y segundo, en qué medida explica la calidad de vida. Por último, se valoró el papel mediador de las estrategias de afrontamiento, considerando que la fuerza de la asociación entre el impacto psicológico y la calidad de vida se ve reducida cuando estas estrategias se incluyen en el modelo de regresión.

Como puede observarse en la Tabla 2, el coeficiente de regresión no estandarizado del impacto psicológico de las condiciones de la piel disminuye de -0.26 en el segundo paso a -0.17 en el tercero, indicando que el $33.84 \%$ de la varianza en la relación impacto psicológico de las condiciones de la piel y calidad de vida se asocia a las estrategias de afrontamiento activadas [(0.26-0.17)/0.26]. Así, se muestra que algunas estrategias de afrontamiento pueden actuar como mediadoras entre las emociones y sentimientos asociados a la percepción de enfermedad dermatológica y la calidad de vida.

Para confirmar la existencia de este efecto mediador se realizó el test de Sobel para cada una de las estrategias de afrontamiento incluidas en el modelo de regresión (Baron \& Kenny, 1986; Sobel, 1982). Para las estrategias Pensamientos desiderativos $(z=3.96, p<0.001)$ y Pensamientos positivos $(z=1.61, p<0.05)$ se obtuvo una reducción estadísticamente significativa de la asociación entre la variable independiente (impacto psicológico de las condiciones de la piel) y la variable dependiente (calidad de vida) al introducir la variable mediadora (estrategias de afrontamiento).

\section{Discusión}

El objetivo de este trabajo ha sido analizar el papel mediador de diferentes estrategias de afrontamiento en el impacto sobre la calidad de vida de la percepción de enfermedad dermatológica de baja expresión en términos de sintomatología y emociones asociadas. La piel es un órgano extenso y visible por lo que es lógico que, cuando está dañado, repercuta en la calidad de vida de las personas afectadas (Badía et al., 1999; Drake et al., 1999; Nichol et al., 1996). También se ha constatado que el afrontamiento es una variable de gran influencia en la percepción de calidad de vida y en la respuesta a la enfermedad (Fortune et al., 2002). 
TABLA 2

Modelos de regresión evaluando el papel mediador de las estrategias de afrontamiento en la relación del impacto psicológico de las condiciones de la piel y la calidad de vida

\begin{tabular}{|c|c|c|c|c|c|c|c|c|c|}
\hline Variable predictora & B & SE & $\beta$ & $\mathrm{t}$ & Tolerancia & FIV & Autovalor & $\begin{array}{l}\text { Índice de } \\
\text { condición }\end{array}$ & $\begin{array}{l}\text { Variable } \\
\text { criterio }\end{array}$ \\
\hline \multicolumn{10}{|l|}{ Paso 1} \\
\hline $\begin{array}{l}\text { Impacto psicológico } \\
R 2=0.26, F(1,218)= \\
76.97 * * * ; D-W=1.8\end{array}$ & 0.91 & 0.1 & 0.51 & $8.77 * * *$ & 1 & 1 & 0.058 & 5.79 & $\begin{array}{c}\mathrm{P} . \\
\text { desiderativos }\end{array}$ \\
\hline $\begin{array}{l}\text { Impacto psicológico } \\
R 2=0.05, F(1,218)= \\
12.33^{* *} ; D-W=1.74\end{array}$ & 0.5 & 0.14 & 0.23 & $3.52 * *$ & 1 & 1 & 0.058 & 5.79 & B. soluciones \\
\hline $\begin{array}{l}\text { Impacto psicológico } \\
R 2=0.04, F(1,218)= \\
10.24^{* *} ; D-W=1.85\end{array}$ & 0.36 & 0.11 & 0.21 & $3.20 * *$ & 1 & 1 & 0.058 & 5.79 & $\begin{array}{l}\text { B. apoyo } \\
\text { social }\end{array}$ \\
\hline $\begin{array}{l}\text { Impacto psicológico } \\
R 2=0.15, F(1,218)= \\
40.74 * * * ; D-W=1.89\end{array}$ & 0.71 & 0.11 & 0.4 & $6.38 * * *$ & 1 & 1 & 0.058 & 5.79 & $\begin{array}{c}\text { B. } \\
\text { información }\end{array}$ \\
\hline $\begin{array}{l}\text { Impacto psicológico } \\
\mathrm{R} 2=0.03, F(1,218)= \\
7.79 * * ; \mathrm{D}-\mathrm{W}=2\end{array}$ & 0.3 & 0.11 & 0.19 & $2.79 * *$ & 1 & 1 & 0.058 & 5.79 & P. positivos \\
\hline $\begin{array}{l}\text { Impacto psicológico } \\
R 2=0.01, F(1,218)=3.52 \\
D-W=1.69\end{array}$ & 0.2 & 0.1 & 0.13 & 1.87 & 1 & 1 & 0.058 & 5.79 & Distanciam. \\
\hline \multicolumn{10}{|c|}{ Paso $2 R 2=0.07, F(1,218)=18.48^{* * *} ; \mathrm{D}-\mathrm{W}=2.06$} \\
\hline Impacto psicológico & -0.26 & 0.06 & -0.28 & $-4.3 * * *$ & 1 & 1 & 0.058 & 5.79 & Calidad vida \\
\hline \multicolumn{10}{|c|}{ Paso $3 R 2=0.16, F(3,216)=13.39 * * * ; D-W=1.95$} \\
\hline Impacto psicológico & -0.17 & 0.07 & -0.18 & $-20.50 * *$ & 0.701 & 1.43 & 0.142 & 7.28 & Calidad vida \\
\hline P. desiderativos & -0.19 & 0.04 & -0.36 & $-4.44 * * *$ & 0.576 & 1.74 & 0.116 & 8.06 & Calidad vida \\
\hline B. soluciones & 0.05 & 0.04 & 0.12 & 1.33 & 0.493 & 2.03 & 0.071 & 10.30 & Calidad vida \\
\hline B. apoyo social & 0.05 & 0.05 & 0.09 & 0.958 & 0.413 & 2.42 & 0.053 & 11.93 & Calidad vida \\
\hline B. información & 0.03 & 0.05 & 0.06 & 0.596 & 0.442 & 2.26 & 0.041 & 13.59 & Calidad vida \\
\hline P. positivos & 0.10 & 0.05 & 0.17 & $2.00 *$ & 0.550 & 1.82 & 0.037 & 14.32 & Calidad vida \\
\hline Distanciamiento & -0.04 & 0.04 & -0.07 & -0.943 & 0.698 & 1.43 & 0.033 & 15.15 & Calidad vida \\
\hline
\end{tabular}

$* p \geq 0.05 . * p \geq 0.01$. *** $p \geq 0.001$.

Fuente: elaboración propia

En línea con otros resultados, se ha observado la importancia de las estrategias de afrontamiento en relación con la calidad de vida (Drake et al., 1999; Mazzotti et al., 2003; Schmid-Ott et al., 2005; Van Loey \& Van Son, 2003).

Sin embargo, una contribución de los resultados de este trabajo ha sido poner de manifiesto que no todas las estrategias de afrontamiento median de igual manera la influencia de la percepción de la enfermedad sobre la calidad de vida. Se ha obtenido que es la estrategia Pensamientos positivos la que contribuye a mantener la calidad de vida, a pesar de que la persona perciba la presencia de daño dermatológico, mientras que la estrategia Pensamientos desiderativos contribuye a disminuir la calidad de vida de los participantes que perciben los mismos problemas. Además, se observa que las estrategias de afrontamiento Búsqueda de soluciones, Búsqueda de apoyo, Búsqueda de información y Distanciamiento no actúan como estrategias de afrontamiento mediadoras de la calidad de vida. Esto pone de manifiesto que realizar conductas dirigidas a obtener ayuda profesional, a lograr la comprensión y apoyo de otras personas, a ampliar el conocimiento del problema o separar el problema de otros ámbitos de la vida no modifica la relación 
entre las consecuencias psicológicas de la enfermedad y la calidad de vida. Cabe destacar que estas estrategias se caracterizan por tener una orientación conductual, a diferencia de las estrategias que sí tienen un papel mediador, que son cognitivas. Quizás esto puede estar relacionado con que las alteraciones dermatológicas estudiadas fueran de baja expresión sintomatológica, de forma que el uso de estrategias cognitivas de afrontamiento puede ser suficiente para relativizar el efecto de la enfermedad sobre la calidad de vida.

Un aspecto que se debe resaltar en este trabajo es que hace referencia a alteraciones dermatológicas de expresión leve, mientras que en la mayoría de los estudios se ha analizado la relación entre la percepción de la enfermedad, la calidad de vida y las estrategias de afrontamiento en personas con enfermedades severas o crónicas (Benyamini et al., 2012; Karademas et al., 2010; Vinaccia et al., 2012). Por lo tanto, una contribución de los resultados obtenidos es que cuando una persona tiene una alteración dermatológica de baja expresión sintomatológica es el impacto psicológico que esta tenga y el uso de determinadas estrategias de afrontamiento lo que va a determinar su calidad de vida. Cuando la patología dermatológica es severa, existen otras variables clínicas relacionadas con la enfermedad que influyen de manera importante en la calidad de vida (Van Loey \& Van Son, 2003).

Estos resultados ponen de manifiesto la utilidad de promover el desarrollo de estrategias de afrontamiento orientadas positivamente hacia el problema no solo en personas con enfermedades severas o crónicas, sino también en aquellas que perciben la presencia de daño dermatológico leve. Si la percepción de la enfermedad, por leve que sea, pone en juego estrategias de afrontamiento más optimistas, la persona valorará de forma más positiva su calidad de vida en general, lo que redundará a su vez en una visión menos negativa de su enfermedad y en un afrontamiento más eficaz.

Una limitación de este estudio tiene que ver con el procedimiento de recogida de datos. Diversos autores han expresado que, cuando quiere realizarse un análisis mediacional, es relevante la secuencia temporal en la medición de las variables implicadas. En estudios sobre enfermedades de baja expresión sintomatológica es complicado realizar este tipo de diseños, porque en muchas ocasiones no hay un diagnóstico temprano de la enfermedad o una percepción clara de la misma. No obstante, en estudios posteriores podría considerarse la posibilidad de tomar medidas sucesivas de las variables de forma que pudiera considerarse el cambio en el tiempo de la relación entre las variables.

En líneas de investigación futuras sería interesante profundizar en la relación bidireccional que puede existir entre las variables consideradas, percepción de la enfermedad, estrategias de afrontamiento y calidad de vida, de forma que pudiera ponderarse la relación circular que pueda existir entre las mismas, así como analizar el efecto que variables sociodemográficas, como el sexo, o características de la enfermedad, como la cronicidad, pudieran tener en esta relación.

También sería interesante estudiar por qué el uso de estrategias de afrontamiento no orientadas al problema, como por ejemplo Pensamientos desiderativos, contribuyen negativamente a la calidad de vida. En este sentido cabría preguntarse si la estrategia contribuye directamente o su efecto sobre la calidad de vida se debe a que bloquea el uso de estrategias de afrontamiento orientadas al problema. También sería interesante incluir en futuros estudios indicadores de las variables analizadas que proporcionen evidencias independientes de los propios participantes.

\section{Referencias}

Badía, X., Mascaró, J. M., \& Lozano, R. (1999). Measuring health-related quality of life in patients with mild to moderate eczema and psoriasis: Clinical validity, reliability and sensitivity to change of the DLQI. British Journal of Dermatology, 141(4), 698-702.

Baron, R. M., \& Kenny, D. A. (1986). The moderatormediator variable distinction in social psychological research: Conceptual, strategic and statistical considerations. Journal of Personality and Social Psychology, 51, 1173-1182. 
Benyamini, Y., Goner-Shilo, D., \& Lazarov, A. (2012). Illness perception and quality of life in patients with contact dermatitis. Contact Dermatitis, 67(4), 193-199.

Burckhardt, C. S., \& Anderson, K. L. (2003). The Quality of Life Scale (QOLS): Reliability, validity, and utilization. Health and Quality of Life Outcomes, 1(1), 60. doi:10.1186/1477-7525-1-60

Burckhardt, C. S., Anderson, K. L., Archenholtz, B., \& Hägg, O. (2003). The Flanagan Quality of Life Scale: Evidence of construct validity. Health and Quality of Life Outcomes, 1(59). doi:10.1186/14777525-1-59

Chren, M. M., Lasek, R. J., Quinn, L. M., Mostow, E. N., \& Zyzanski, S. J. (1996). Skindex, a quality-of-life measure for patients with skin disease: Reliability, validity, and responsiveness. Journal of Investigative Dermatology, 107, 707-713.

Delhey, J. (2004). Life satisfaction in an enlarged Europe. Luxembourg: Office for Official Publications of the European Communities. Eurofound. Recuperado el de http://www.eurofound.europa.eu/publications/htmlfiles/ef03108.htm

Dijkers, M. (2005). Quality of life of individuals with spinal cord injury: A review of conceptualization, measurement, and research findings. Journal of Rehabilitation Research EO Development, 42(3), 87-110.

Drake, L. A., Patrick, D. L., Fleckman, P., André, J., Baran, R., Haneke, E., ... Tosti, A. (1999). The impact of onychomycosis on quality of life: Development of an international onychomycosis-specific questionnaire to measure patient quality of life. Journal of the American Academy of Dermatology, 41(2), 189-196.

Flanagan, J. C. (1978). A research approach to improving our quality of life. American Psychologist, 33, 138-147.

Fortune, D. G., Richards, H. L., Griffih, E. M., \& Main, C. J. (2002). Psychological stress, distress and disability in patients with psoriasis: Consensus and variation in the contribution of illness perceptions, coping and alexithymia. British Journal of Clinical Psychology, 41(2), 157-174.

Gupta, M. A., Gupta, A. K., \& Watteel, G. N. (1997). Stress and alopecia areata: A psychodermatologic study. Acta Dermato-Venereology, 77(4), 296-298.
Hernández-Fernaud, E., Hernández, B., Ruiz, A., Rodríguez, A., \& Betancor, V. (2008). Impacto de las alteraciones dermatológicas de baja expresión en función del nivel de especificidad de la calidad de vida. Psicothema, 20 (2), 273-278.

Hernández-Fernaud, E., Hernández, B., Ruiz, C., \& Ruiz, A. (2009). Development of a questionnaire to evaluate coping strategies for skin problems. Spanish Journal of Psychology, 12(1), 373-382.

Jones-Caballero, M., Penas, P. F., García-Diez, A., Chren, M. M., \& Badía, X. (2002). La versión española de Skindex-29. Un instrumento de medida de la calidad de vida en pacientes con enfermedades cutáneas. Medicina Clínica, 118(1), 5-9.

Judd, C. M., \& Kenny, D. (1981). Process analysis: Estimating mediation in treatment evaluations. Evaluation Review, 5, 602-619.

Judd, C. M., \& Kenny, D. (2010). Data analysis in social psychology: Recent and recurrent issues. En S. T. Fiske, D. T. Gilbert \& G. Lindzey (Eds.), Handbook of social psychology (5.a ed.) (pp. 115-139). New York: Wiley.

Karademas, E. C., Zarogiannos, A., \& Karamvakalis, N. (2010). Cardiac patient-spouse dissimilarities in illness perception: Associations with patient self-rated health and coping strategies. Psychology and Health, 25(4), 451-463.

Latorre-Román, P. A., Martínez-Amat, A., MartínezLópez, E., Moral, A., Santos, M. A., \& HitaContreras, F. (2014).Validation and psychometric properties of the Spanish version of the Quality of Life Scale (QOLS) in patients with fibromialgia. Rheumatology International, 34(4), 543-549. doi: 10.1007/s00296-013-2874-1

Lazarus, R. S. (1993). From psychological stress to the emotions: A history of changing outlooks. Annual Reviews of Psychology, 44, 1-21.

Lazarus, R. S., \& Folkman, S. (1986). Estrés y procesos cognitivos. Barcelona: Martínez Roca. (Trabajo original publicado en 1984)

Leventhal, H., Leventhal, E. A., \& Cameron, L. (2001). Representations, procedures, and affect in illness self-regulation: A perceptual-cognitive model. En A. Baum, T. A. Revenson \& J. E. Singer (Eds.), Handbook of health psychology (pp. 19-48). Mahwah, NJ: Erlbaum. 
Mazzotti, E., Picardi, A., Sampogna, F., Sera, F., Pasquín, P., \& Abeni, D. (2003). Sensitivity of the Dermatology Life Quality Index to clinical change in patients with psoriasis. British Journal of Dermatology, 149, 318-322.

Nava-Quiroz, C., Vega-Valero, C., \& Soria, R. (2010). Escala de modos de afrontamiento: consideraciones teóricas y metodológicas. Universitas Psychologica, 9(1), 139-147.

Nichol, M. B., Margolis, J. E., Lippa, E., Rower, M., \& Quell, J. (1996). The application or multiple quality of life instruments in individuals with mildtwo-moderate psoriasis. Pharmacoeconomics, 10, 644-653.

Niemeier, V., Kupfer, J., Demmelbauer-Ebner, M., Stangier, U., Effendy, I., \& Gieler, U. (1998). Coping with acne vulgaris. Dermatology, 196, 108-115.

Pantiga, C., López, L., Pérez, M., Rodríguez, M., Linares, A., Dieguez, L. G., ... Rodrigo, L. (2005). Quality of life in cirrhotic patients and liver transplant recipients. Psicothema, 17(1), 143-147.

Rapp, S. R., Cottrell, C. A., \& Leary, M. R. (2001). Social coping strategies associated with quality of life decrements among psoriasis patients. British Journal of Dermatology, 145(4), 610-616.

Revicki, D. A., Osoba, D., Fairclough, D., Barofsky, I., Berzon, R., Leidy, N. K., \& Rothman, M. (2000). Recommendations on health-related quality of life research to support labeling and promotional claims in the United States. Quality Life Research, 9(8), 887-900.

Rodríguez-Marín, J., Pastor, A., \& López-Roig, S. (1993). Afrontamiento, apoyo social, calidad de vida y enfermedad. Psicothema, 5(Supl.), 349-372.

Rubino, I. A., Sonnino, A., Pezzarossa, B., Ciani, N., \& Bassi, R. (1995). Personality disorders and psychiatric symptoms in psoriasis. Psychological Reports, 77(2), 547-553.

Sampogna, S., Sera, F., Aveni, D., \& Istituto Dermopatico dell'Immacolata Multipurpose Psoriasis Research on Vital Experiences investigators. (2004).
Measures of clinical severity, quality of life, and psychological distress in patients with psoriasis: A cluster analysis. Journal of Investigative Dermatology, 122(3), 602-607.

Scharloo, M., Kaptein, A. A., Weinman, J., Bergman, W., Vermeer, B. J., \& Rooijmans, H. G. (2000). Patients' illness perceptions and coping as predictors of functional status in psoriasis: A 1-year follow-up. British Journal of Dermatology, 142(5), 899-907.

Schmid-Ott, G., Künsebeck, H. -W., Jäger, B., Sittig, U., Hofste, N., Ott, R., ... Lamprecht, F. (2005). Significance of the stigmatization experience of psoriasis patients: A 1-year follow-up of the illness and its psychosocial consequences in men and women. Acta Dermato-Venereologica, 85(1), 27-32.

Schmidt, S., Fischer, T. W., Chren, M. M., Strauss, B. M., \& Elsner, P. (2001). Strategies of coping and quality of life in women with alopecia. British Journal of Dermatology, 144(5), 1038-1043.

Sobel, M. E. (1982). Asymptotic confidence intervals for indirect effects in structural equation models. Sociological Methodology, 13, 290-312.

Tabachnick, B. G., \& Fidell, L. S. (1989). Using multivariate statistics (2.. ed.). New York: Harper Collins.

Van Loey, N., \& Van Son, M. (2003). Psychopathology and psychological problems in patients with burn scars. American Journal of Clinical Dermatology, 4(4), 245-272.

Vinaccia, S., Quiceno, J. M., \& Remor, E. (2012). Resiliencia, percepción de enfermedad, creencias y afrontamiento espiritual-religioso en relación con la calidad de vida relacionada con la salud en enfermos crónicos colombianos. Anales de Psicología, 28(2), 366-377.

Wahl, A., Hanestad, B. R., Wiklund, I., \& Moum, T. (1999). Coping and quality of life in patients with psoriasis. Quality of Life Research, 8:(5), 427-433.

Zeller, R. A., \& Carmines, E. G. (1980). Measurement in the social sciences. New York: Cambridge University Press. 
